\title{
Methylenetetrahydrofolate Reductase A1298C Polymorphism and Major Depressive Disorder
}

Kevin Cho ${ }^{1}$, Zubair M. Amin ${ }^{2}$, Jie An ${ }^{3}$, Kerry Anne Rambaran ${ }^{4}$, Tyler B. Johnson ${ }^{3}$, Saeed K. Alzghari $^{3}$

1. Reference Health Laboratories, Gulfstream Diagnostics 2. Thomas J. Long School of Pharmacy \& Health Sciences, University of the Pacific 3. Gulfstream Genomics, Gulfstream Diagnostics 4. Department of Clinical Sciences, Keck Graduate Institute

$\square$ Corresponding author: Saeed K. Alzghari, salzghari@gulfdiagnostics.com Disclosures can be found in Additional Information at the end of the article

\section{Abstract}

Major depressive disorder (MDD) is a disorder that carries significant psychosocial and economic implications. Research efforts have focused on identifying biomarkers that can aid in the prediction, diagnosis, and efficacious treatment of MDD. Most of this focus has been placed on a polymorphism of the methylenetetrahydrofolate reductase (MTHFR) gene, C677T. MTHFR C677T is screened during MDD diagnosis in many protocols. However, MTHFR C667T poses conflicting data in various ethnic groups and geographic populations calling into question its utility. Another polymorphism, MTHFR A1298C, has often taken the back-seat to MTHFR C677T in respect to research focus. MTHFR A1298C is implicated in irregular homocysteine metabolism and aberrant folate cycles and, through this, it may play a role as either a driver in the development of MDD or as a predictive or diagnostic marker, possibly in combination with C677T. The number of studies evaluating MTHFR A1298C and the power of those studies is lacking and thus larger studies are required to confirm the association between this polymorphism and MDD.

Categories: Genetics, Psychiatry, Healthcare Technology

Keywords: depression, homocysteine, single nucleotide polymorphisms, methylenetetrahydrofolate reductase, polymorphism, mthfr, major depressive disorder, a1298c

\section{Introduction And Background}

Received 09/21/2017 Review began 09/25/2017 Review ended 09/26/2017 Published 10/01/2017

C) Copyright 2017

Cho et al. This is an open access article distributed under the terms of the Creative Commons Attribution License CC-BY 3.0., which permits unrestricted use, distribution, and reproduction in any medium, provided the original author and source are credited.
Major depressive disorder (MDD) or clinical depression, is a mental disorder characterized by a persistent feeling of hopelessness or despair and a general loss of interest in daily activities. Depression can have a significant impact on a person's well-being, often negatively affecting one's daily behavior, sleeping habits, self-esteem, or energy levels [1]. In 2015, nearly 16 million adults in the United States (U.S.) had experienced MDD within the previous year, and this number accounts for approximately $7 \%$ of all adults in the U.S. [2]. Given the prevalence and socioeconomic implications of depression, greater research emphasis is being placed on the enigmatic and complex biochemical reactions that underlie depression. Some researchers, like a group at Harvard, have sought to understand links between the slow production of new neurons and periods of depression. Interestingly, these conclusions might help to explain why there is a delayed reaction of several weeks when some individuals undergo treatment with antidepressants [3]. Other groups have begun to explore connections between genetic polymorphisms in genes such as methylenetetrahydrofolate reductase (MTHFR) and an individual's propensity towards major depression. The MTHFR gene encodes the enzyme called 


\section{Cureus}

methylenetetrahydrofolate reductase (MTHFR). This enzyme plays an important role in the folate-cycle through the conversion of 5, 10-methylenetetrahydrofolate to 5methyltetrahydrofolate (5-MTHF). 5-MTHF will then donate a methyl group in the conversion of homocysteine (HCY) to methionine [4]. A lack of MTHFR or expression reduced-function variant of the enzyme will lead to decreased levels of 5-methyltetrahydrofolate, thus resulting in high levels of HCY. Both irregular folate metabolism and high levels of HCY have shown to be correlated with MDD and may be indicators of polymorphisms in the MTHFR gene [5-7]. Furthermore, there is evidence that has suggested both the MTHFR C677T (dbSNP: rs1801133) and A1298C (dbSNP: rs1801131) single nucleotide polymorphisms (SNPs) are correlated to MDD and its treatment [7-9]. While much research focus has been placed on MTHFR C677T as the primary polymorphism of concern, recent mounting evidence also implicates A1298C as another important polymorphism of the MTHFR gene. In the following review, we seek to highlight evidence of the single nucleotide polymorphism, MTHFR A1298C, in the context of MDD.

\section{Review}

The aim of this review was to assess the MTHFR A1298C polymorphism and its relationship to MDD. A literature search for articles in PubMed, ISI Web of Knowledge, and Scopus was performed. The terms for the search strategy were "MTHFR," “A1298C," and “depression.” We utilized the following criteria for article selection: 1) articles written in English; 2) must be fulltext articles; and 3) must be either a randomized controlled trial, prospective trial, retrospective analysis, case series, or case report. We examined the reference lists of each article for additional resources that fit the selection criteria. We initially identified 15 articles. We excluded an article related to bipolar disorder, an article that failed to isolate depression patients' MTHFR status, an article related to ophthalmology, five meta-analyses, and one review article. In total, six relevant articles were identified for this review and we summarize our findings in Table 1 [6-11].

\begin{tabular}{|c|c|c|c|c|}
\hline $\begin{array}{l}\text { Author } \\
\text { (Year) }\end{array}$ & Population & Intervention & Control & Outcome \\
\hline $\begin{array}{l}\text { Reif et al. } \\
\text { (2005) [8] }\end{array}$ & $\begin{array}{l}\text { Female inpatients with } \\
\text { acute psychiatric disorders } \\
(n=120) \text { Bipolar disorder } \\
\text { and MDD inpatients in } \\
\text { Germany }(n=136)\end{array}$ & $\begin{array}{l}\text { - Genotyping } \\
\text { SNPs: C677T \& } \\
\text { A1298C } \\
\text { - Determination of } \\
\text { HCY, vitamin } \\
\text { B12, \& folate } \\
\text { levels }\end{array}$ & $\begin{array}{l}\text { Healthy blood } \\
\text { donors from } \\
\text { the same } \\
\text { region with } \\
\text { low chance of } \\
\text { MDD (n=284) }\end{array}$ & $\begin{array}{l}\text { - } \text { Severity } \\
\text { associated with } \\
\text { high HCY level } \\
\text { - } \text { Elevated HCY } \\
\text { associated with } \\
\text { elderly* } \\
\text { A1298C CC } \\
\text { genotype } \\
\text { associated with } \\
\text { MDD and bipolar } \\
\text { disorder* }\end{array}$ \\
\hline Evinova & & $\begin{array}{l}\text { - Genotyping SNPs } \\
\text { associated with } \\
\text { MDD: G196A, }\end{array}$ & $\begin{array}{l}\text { - Healthy blood } \\
\text { donors without }\end{array}$ & $\begin{array}{l}\text { A1298C: Allele C } \\
\text { frequency } \\
\text { associated with } \\
\text { 39.2\% MDD and }\end{array}$ \\
\hline
\end{tabular}




\section{Cureus}

et al. Patients with MDD $(n=134)$

(2012) [9]
Jamerson Elderly patients (age $>60$ )

et al.

(2013)

[11] with MDD who are prescribed with SSRI ( $n=104)$
Patients enrolled in Diamond Study with MDD $(n=147)$
C677T, \&

A1298C

$\operatorname{MDD}(\mathrm{n}=143)$

- Baseline

measures of

- DSM, CES-D, \& PHQ severity assessment at various time intervals $(\mathrm{t}=0$, $24,36,48, \& 60$ months)

- MADRS severity assessment at every 3 months for remission determination

- Genotyping 15 SNPs from 10 genes from folate metabolism

- Folate intake estimated via

Block 1998

Questionnaire
- SSRI treatments

- Folate intake
- A1298C AC

genotype associated with 2.5x likelihood with SSRI treatment*
- A1298C CC genotype more frequent in female MDD

- Genotyping MTHFR and COMT - Healthy polymorphism: patients than controls*

- A1298C significant
$29.7 \%$ control; more risk for MDD* between A1298C and MDD prognosis 


\section{Cureus}

Nielsen et Patients with MDD who

al. (2015) were resistant $(n=389)$ and

[6]

responsive $(n=224)$ to

antidepressants

Mech et Patients with positive

al. (2016) C677T and A1298C SNPs

[7] $\quad(n=159)$
C677T, A1298C, \& Val158Met
- cDNA synthesis

$$
\begin{aligned}
& \text { volunteers } \\
& \text { without MDD } \\
& (\mathrm{n}=463)
\end{aligned}
$$
from fibroblasts RNA$$
\text { and allele } \mathrm{A}
$$$$
\text { carriers* }
$$

- A1298C CC genotype associated with lower age of onset

- Reduction in

- Administration of EnLyte ${ }^{\circledR}$ (reduced B vitamins)

- MADRS severity at various time interval $(\mathrm{t}=0,2$, \& 8 weeks)
- Positive C677T and A1298C SNPS

- Administration of placebo $(\mathrm{n}=123)$

- $\operatorname{MADRS}(\mathrm{t}=0)$ homocysteine

levels at week 8

in $82.4 \%$ of

active treatment patients*

- Full remission achieved by week 8 in $42 \%$ of active treatment patients*

\section{TABLE 1: PICO analysis of current MTHFR A1298C major depressive disorder studies}

*Statistically significant $(p \leq 0.05)$

Abbreviations: cDNA- complementary deoxyribonucleic acid; CES-D- Center for Epidemiologic Studies Depression Scale; COMTcatechol-O-methyl transferase; DSM- Diagnostic and Statistical Manual of Mental Disorders; MDD- major depressive disorder; HCY- homocysteine; htSNP- haplotype tagging single nucleotide polymorphism; MADRS- Montgomery-Åsberg Depression Rating Scale; MTHFR- methylenetetrahydrofolate reductase; PHQ- Patient Health Questionnaire; RNA- ribonucleic acid; SNP- single nucleotide polymorphism; SSRI- selective serotonin reuptake inhibitor

Note: All major depressive disorder patients were diagnosed by DSM-IV or DSM-V criteria

\section{A1298C and risk of depression}

A transversion at nucleotide 1298 in exon 7 on the MTHFR gene leads to a $60 \%$ reduction in enzyme activity compared to the wild-type enzyme [12]. While the C677T transition does lead to greater reduction of the enzymatic activity of MTHFR than that of A1298C, the current findings on C677T alone are conflicting and limit association between the genotype and its implication in MDD [13-16]. A few recent studies examined the association of both C677T and A1298C over the same population that may alleviate the aforementioned conflicts. By genotyping 136 female patients and 284 controls in a study by Reif, et al., A1298C was determined to be significantly associated with both MDD and bipolar disorder whereas C677T 
failed to find a similar association [8]. Another study by Evinova, et al. enrolling 143 patients and 134 controls showed that $\mathrm{C}$ allelic frequency and CC genotype male carriers were significantly associated with MDD incidence [9]. These two studies by Reif, et al. and Evinova, et al. delve into genetic variations in the Caucasian population, specifically German and Slovak ancestries, respectively, in which the prevalence in polymorphisms of the CC genotype in A1298C (8.54\%) is lower than the TT genotype in C677T (12.5\%) [11]. Additionally, the TT genotype of C677T appears to have modest effect on the risk of MDD [10, 13, 17]. A recent study by Nielsen and co-workers on A1298C failed to find the same association between A1298C and MDD despite assessing a large population of 613 patients and 463 controls [6]. The study, however, additionally noted that the strong association was found only in female patients and controls with higher statistical power than the previous studies. Based on these findings, A1298C has the potential to be an alternative or complementary, gender-specific indicator in diagnosing MDD in the Caucasian population. However, additional studies with a larger population size are needed to validate this.

Other studies investigated the association between the A1298C genotypes and MDD prognosis. In one small study by Bousman et al. with variable treatment modality, A1298C did not show any association between its genotypes and MDD prognosis, whereas C677T CC genotype had greater severity scores and indicated poor remission over 60 months [10]. However, Jamerson et al. found that the A1298C AC genotype is significantly more likely to be in remission as compared with the A1298C AA genotype when subjected to selective serotonin reuptake inhibitor (SSRI) treatments [11]. While the MTHFR gene has the potential to predict MDD prognosis by assessing both SNPs, their strong correlation yet remains to be elucidated. Further, these studies suggest that the prognosis may not depend solely on reduced enzyme activity. Alternatively, they suggest C677T and A1298C may affect HCY level to varying degree.

\section{A1298C, diet, and homocysteinemia}

MTHFR regulates the conversion of HCY to methionine through catalyzing the formation of 5MTHF. Reduced enzyme activity through function-reducing polymorphisms results in the impairment of HCY metabolism and the folate cycle. Impairment of these processes results in the inadequate synthesis of serotonin and myelin as well as aberrations in other neural and vascular pathways [18]. Only a few studies have investigated the link between A1298C and HCY metabolism, yielding conflicting results [19-23].

In the study by Jamerson, et al., nutritional folate intake in a geriatric population was monitored along with genetic sampling for genes associated with folate metabolism [11]. The study demonstrated a slight increase in mean total folate level in the non-remitting population but no robust association. Similarly, serum folate level was normal in all cases, although 16 out of 24 patients had elevated HCY in the study performed by Reif, et al. [8]. Based on these findings, dietary folate intake does not appear to affect the level of HCY or predisposition of depression. However, elevated HCY levels were still observed in the same population that may suggest dietary folate conversion to the co-substrate is impaired. In a recent study by Mech and Farah, this idea was investigated by administering the metabolized vitamins and micronutrients in a randomized double-blind study [7]. Mech and Farah showed that $82.4 \%$ of 159 patients with a positive C677T or A1298C polymorphism demonstrated a reduction in HCY levels when treated with reduced vitamin B while 123 controls demonstrated a slight increase in HCY from baseline, which was statistically significant [7]. Because their study used a population that included either SNP, HCY elevation due to A1298C could not be accurately predicted. Furthermore, it was found in previous studies that A1298C has a significant effect on enzyme activity, but was not strongly associated with increased HCY concentrations [12].

In addition, Mech and Farah showed that $42 \%$ of patients with either an MTHFR A1298C or C677T polymorphism achieved full remission by week eight compared to the control population 
that was given placebo, while other studies that included elderly patients agree with similar associations between HCY level and MDD by noting that HCY level is strongly associated with age [7]. Despite the controlled folate intake, vascular dysfunction manifests into psychiatric symptoms which place the elderly population more at risk for poorer outcomes related to diet and genetic differences in folate genes [8, 11, 24].

\section{Limitations}

The primary limitation to A1298C analysis is the lack of large studies of high statistical power. Furthermore, a dearth of randomized controlled trials on this topic exist and most of the data presented are from observational studies. Although the aforementioned trials may not have been powered, many of the A1298C studies showed statistical significance associated with depression risk and homocysteinemia that are worth evaluating in larger trials.

\section{Future directions}

Future A1298C studies should examine large populations in various ethnicities and geographic locations. Diagnostic and Statistical Manual of Mental Disorders, Fifth Edition (DSM-V) and the anticipated International Classification of Diseases (ICD)-11 should continue to give researchers proper guidance in selecting such populations for study. Due to subjectivity, a combination of rating scales should be administered to assess the remission of MDD such as the Montgomery-Åsberg Depression Rating Scale (MADRS), the Patient Health Questionnaire-9 (PHQ-9), the Center for Epidemiologic Studies Depression Scale (CES-D), or the Composite International Diagnostic Interview (CIDI). Additionally, more randomized studies should focus on solely examining the influence of A1298C instead of both A1298C and C677T to better isolate the influence of A1298C. Finally, according to a retrospective cross-sectional study evaluating Medicare claims, a total of 182,358 MTHFR tests were billed in 2013 [25]; thus, a consideration of A1298C as part of a larger decision algorithm for clinicians currently utilizing the MTHFR gene in practice should be incorporated as an option for patients with MDD [26].

\section{Conclusions}

Based on current studies, the MTHFR A1298C polymorphism has the potential to be an alternative or complementary gender-specific indicator in MDD diagnosis, but requires further study. The role of A1298C either by itself or in combination with MTHFR C677T in MDD prognosis must be further elucidated. Additionally, the comparative measures of the likelihood of MDD between the two polymorphisms must also be examined.

\section{Additional Information \\ Disclosures}

Conflicts of interest: In compliance with the ICMJE uniform disclosure form, all authors declare the following: Payment/services info: All authors have declared that no financial support was received from any organization for the submitted work. Financial relationships: Kevin Cho, Jie An, Tyler B. Johnson, and Saeed K. Alzghari declare(s) employment from Gulfstream Diagnostics. Other relationships: All authors have declared that there are no other relationships or activities that could appear to have influenced the submitted work.

\section{References}

1. Depression. (2016). Accessed: September 20, 2017: https://www.nimh.nih.gov/health/topics/depression/index.shtml.

2. Major depression among adults. (2015). Accessed: September 20, 2017: https://www.nimh.nih.gov/health/statistics/prevalence/major-depression-among- 
adults.shtml.

3. What causes depression?. (2017). Accessed: September 20, 2017:

https://www.health.harvard.edu/mind-and-mood/what-causes-depression.

4. MTHFR gene. (2017). Accessed: September 20, 2017: https://ghr.nlm.nih.gov/gene/MTHFR.

5. Fraguas R Jr, Papakostas GI, Mischoulon D, Bottiglieri T, Alpert J, Fava M: Anger attacks in major depressive disorder and serum levels of homocysteine. Biol Psychiatry. 2006, 60:270274. 10.1016/j.biopsych.2005.08.026

6. Nielsen MG, Congiu C, Bortolomasi M, et al.: MTHFR: genetic variants, expression analysis and COMT interaction in major depressive disorder. J Affect Disord. 2015, 183:179-186. 10.1016/j.jad.2015.05.003

7. Mech AW, Farah A: Correlation of clinical response with homocysteine reduction during therapy with reduced B vitamins in patients with MDD who are positive for MTHFR C677T or A1298C polymorphism: a randomized, double-blind, placebo-controlled study. J Clin Psychiatry. 2016, 77:668-671. 10.4088/JCP.15m10166

8. Reif A, Pfuhlmann B, Lesch KP: Homocysteinemia as well as methylenetetrahydrofolate reductase polymorphism are associated with affective psychoses. Prog Neuropsychopharmacol Biol Psychiatry. 2005, 29:1162-1168. 10.1016/j.pnpbp.2005.06.027

9. Evinova A, Babusikova E, Straka S, Ondrejka I, Lehotsky J: Analysis of genetic polymorphisms of brain-derived neurotrophic factor and methylenetetrahydrofolate reductase in depressed patients in a Slovak (Caucasian) population. Gen Physiol Biophys. 2012, 31:415-422. 10.4149/gpb_2012_049

10. Bousman CA, Potiriadis M, Everall IP, Gunn JM: Methylenetetrahydrofolate reductase (MTHFR) genetic variation and major depressive disorder prognosis: a five-year prospective cohort study of primary care attendees. Am J Med Genet B Neuropsychiatr Genet. 2014, 165:68-76. 10.1002/ajmg.b.32209

11. Jamerson BD, Payne ME, Garrett ME, Ashley-Koch AE, Speer MC, Steffens DC: Folate metabolism genes, dietary folate and response to antidepressant medications in late-life depression. Int J Geriatr Psychiatry. 2013, 28:925-932. 10.1002/gps.3899

12. Lievers KJ, Boers GH, Verhoef $\mathrm{P}$, et al.: A second common variant in the methylenetetrahydrofolate reductase (MTHFR) gene and its relationship to MTHFR enzyme activity, homocysteine, and cardiovascular disease risk. J Mol Med (Berl). 2001, 79:522-528. 10.1007/s001090100253

13. Zintzaras E: C677T and A1298C methylenetetrahydrofolate reductase gene polymorphisms in schizophrenia, bipolar disorder and depression: a meta-analysis of genetic association studies. Psychiatr Genet. 2006, 16:105-115. 10.1097/01.ypg.0000199444.77291.e2

14. Gaysina D, Cohen S, Craddock N, et al.: No association with the 5,10methylenetetrahydrofolate reductase gene and major depressive disorder: results of the depression case control (DeCC) study and a meta-analysis. Am J Med Genet B Neuropsychiatr Genet. 2008, 147B:699-706. 10.1002/ajmg.b.30665

15. López-León S, Janssens AC, González-Zuloeta Ladd AM, Del-Favero J, Claes SJ, Oostra BA, van Duijn CM: Meta-analyses of genetic studies on major depressive disorder . Mol Psychiatry. 2008, 13:772-785. 10.1038/sj.mp.4002088

16. Peerbooms OL, van Os J, Drukker M, et al.: Meta-analysis of MTHFR gene variants in schizophrenia, bipolar disorder and unipolar depressive disorder: evidence for a common genetic vulnerability?. Brain Behav Immun. 2011, 25:1530-1543. 10.1016/j.bbi.2010.12.006

17. Wu AH, Kearney T: Lack of impairment due to confirmed codeine use prior to a motor vehicle accident: role of pharmacogenomics. J Forensic Leg Med. 2013, 20:1024-1027. 10.1016/j.jflm.2013.09.019

18. Paul RT, McDonnell AP, Kelly CB: Folic acid: neurochemistry, metabolism and relationship to depression. Hum Psychopharmacol. 2004, 19:477-488. 10.1002/hup.614

19. Friedman G, Goldschmidt N, Friedlander Y, et al.: A common mutation A1298C in human methylenetetrahydrofolate reductase gene: association with plasma total homocysteine and folate concentrations. J Nutr. 1999, 129:1656-1661.

20. Dekou V, Whincup P, Papacosta O, et al.: The effect of the C677T and A1298C polymorphisms in the methylenetetrahydrofolate reductase gene on homocysteine levels in elderly men and women from the British regional heart study. Atherosclerosis. 2001, 154:659-666. 10.1016/S0021-9150(00)00522-0

21. Kumar J, Das SK, Sharma P, Karthikeyan G, Ramakrishnan L, Sengupta S: Homocysteine levels 


\section{Cureus}

are associated with MTHFR A1298C polymorphism in Indian population. J Hum Genet. 2005, 50:655-663. 10.1007/s10038-005-0313-1

22. Klai S, Fekih-Mrissa N, El Housaini S, Kaabechi N, Nsiri B, Rachdi R, Gritli N: Association of MTHFR A1298C polymorphism (but not of MTHFR C677T) with elevated homocysteine levels and placental vasculopathies. Blood Coagul Fibrinolysis. 2011, 22:374-378.

10.1097/MBC.0b013e328344f80f

23. Zappacosta B, Graziano M, Persichilli S, Di Castelnuovo A, Mastroiacovo P, Iacoviello L: 5,10Methylenetetrahydrofolate reductase (MTHFR) C677T and A1298C polymorphisms: genotype frequency and association with homocysteine and folate levels in middle-southern Italian adults. Cell Biochem Funct. 2014, 32:1-4. 10.1002/cbf.3019

24. Alexopoulos GS, Meyers BS, Young RC, Campbell S, Silbersweig D, Charlson M: 'Vascular depression' hypothesis. Arch Gen Psychiatry. 1997, 54:915-922.

10.1001/archpsyc.1997.01830220033006

25. Lynch JA, Berse B, Dotson WD, Khoury MJ, Coomer N, Kautter J: Utilization of genetic tests: analysis of gene-specific billing in Medicare claims data. Genet Med. 2017, 19:890-899. 10.1038/gim.2016.209

26. Alzghari SK, Blakeney L, Rambaran KA: Proposal for a pharmacogenetic decision algorithm . Cureus. 2017, 9:e1289. 10.7759/cureus.1289 